F. Maloberti, E. Bonizzoni, A. Surano: "Time variant digital sigma-delta modulator for fractional-N frequency synthesizers"; IEEE International Symposium on Radio-Frequency Integration Technology, RFIT 2009, Singapore, 9-11 December 2009, pp. 111-114.

(C)20xx IEEE. Personal use of this material is permitted. However, permission to reprint/republish this material for advertising or promotional purposes or for creating new collective works for resale or redistribution to servers or lists, or to reuse any copyrighted component of this work in other works must be obtained from the IEEE. 


\title{
Time Variant Digital Sigma-Delta Modulator for Fractional-N Frequency Synthesizers
}

\author{
Franco Maloberti, Edoardo Bonizzoni, and Antonio Surano \\ Department of Electronics, University of Pavia \\ Via Ferrata, 1 - 27100 Pavia - ITALY \\ E-mail: [franco.maloberti, edoardo.bonizzoni, antonio.surano]@unipv.it
}

\begin{abstract}
A digital sigma-delta modulator whose noise transfer function changes randomly in time is presented. The spectrum of the output bit-stream is tone free for any constant input value. The time-variant behaviour causes a white floor in the noise output spectrum, but the effect is negligible if the levels is kept well below other white noise sources. The scheme preserves the high frequency phase noise even at critical inputs. The hardware cost is affordable and the system complexity is lower than using dither.
\end{abstract}

\section{INTRODUCTION}

Fractional-N synthesizers are important blocks widely used in modern communication systems. The frequency synthesizer is required to ensure low phase noise, good loop speed, limited spurs level, along with fine-grained resolution (channel spacing) at any fractional value, [1-2].

In synthesizers, the Voltage-Controlled Oscillator (VCO) frequency is divided by a number that varies around a fixed integer value $N$ by a variable amount $\Delta N(t)$. The parameter $\Delta N(t)$ is the result of the conversion of the fractional part given by a digital sigma-delta $(\Sigma \Delta)$. The aim of the $\Sigma \Delta$ is to avoid repetitive patterns in the time domain because they give spurious tones, [3]. The designer obtains the result with high order schemes because many integrators and multiple feedback loops suitably scramble the data. However, for critical values of the fractional input even high order architectures show tones in the output spectrum that are removed by dithering, [4].

Unfortunately, non-linearities reduce the effectiveness of dithering. Often, even with a dither that completely removes tones and obtains a well shaped noise spectrum, the phase noise is not good because of the non-linearity of various blocks, like the dead-zone of the charge pump. The nonlinearity mixes the tones not removed by the loop filter and worsen the phase noise contributed by the $\Sigma \Delta$ in frequency regions where the other noise terms are negligible.

This paper proposes an effective method for destroying the repetitive patterns of time-domain $\Sigma \Delta$ signals. Instead of using a noise-like term that is added to signals (dither), a noise-lke additive term for the modulator coefficients is used. Simulations at the behavioural level show that dithering in multiplication instead of in addition is more effective. The phase noise performance remains remarkable even with nonlinearities in the synthesizer loop.

\section{Conventional Solutions}

The basic block diagram of a conventional fractional-N synthesizer is shown in Fig. 1. The Phase Frequency Detector (PFD) receives the reference clock and the VCO divided by $N+\Delta N(t)$. The loop is then closed by the charge pump and loop filter. The main designer concerns are to ensure a low phase noise and avoid spurs in the output signal. The noise sources affecting the blocks of Fig. 1 are the $\Sigma \Delta$ noise, the PFD, the charge pump, the reference clock and the VCO noise. Since these noise sources are not correlated, they contribute quadratically to the overall phase noise

$$
P N_{T}^{2}=P N_{\Sigma \Delta}^{2}+P N_{P F D}^{2}+P N_{C P}^{2}+P N_{\text {Clock }}^{2}+\ldots ;
$$

The power spectra of every single component, assuming a linear response, are summed up.

The undesired in-band spurious tones are typically caused by the $\Sigma \Delta$ because, with critical fractional values input, it gives rise to limit cycles in the time domain, [5-6].

Since the digital $\Sigma \Delta$ is required to produce low noise at low frequencies and no spurs, the order of the modulator must be at least 3 with the spurs possibly cancelled by dithering. High order modulators are the right choice because they are able obtain the conditions for which the quantization error is equivalent to noise. However, hidden tones in the high frequency region of the spectrum create problems.

Notice that having a strongly shaped $\Sigma \Delta$ noise is not relevant because at low frequencies other sources of noise are

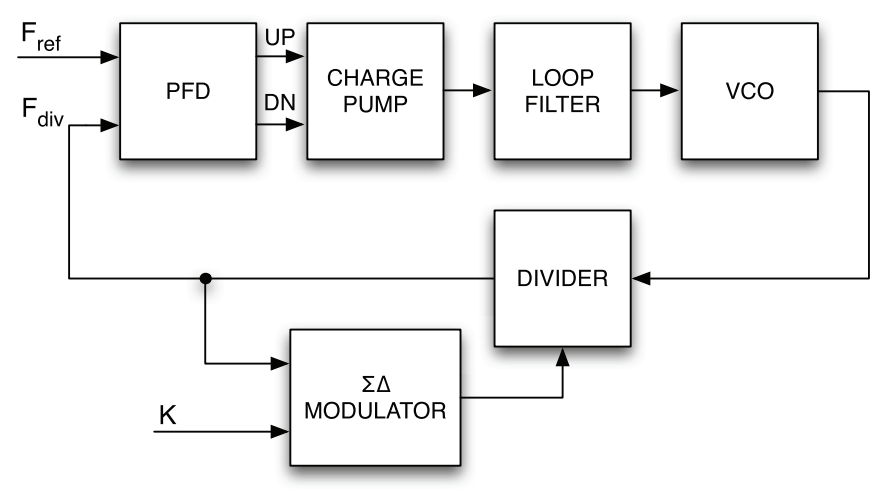

Fig. 1. Fractional-N frequency sinthesizer block diagram. 


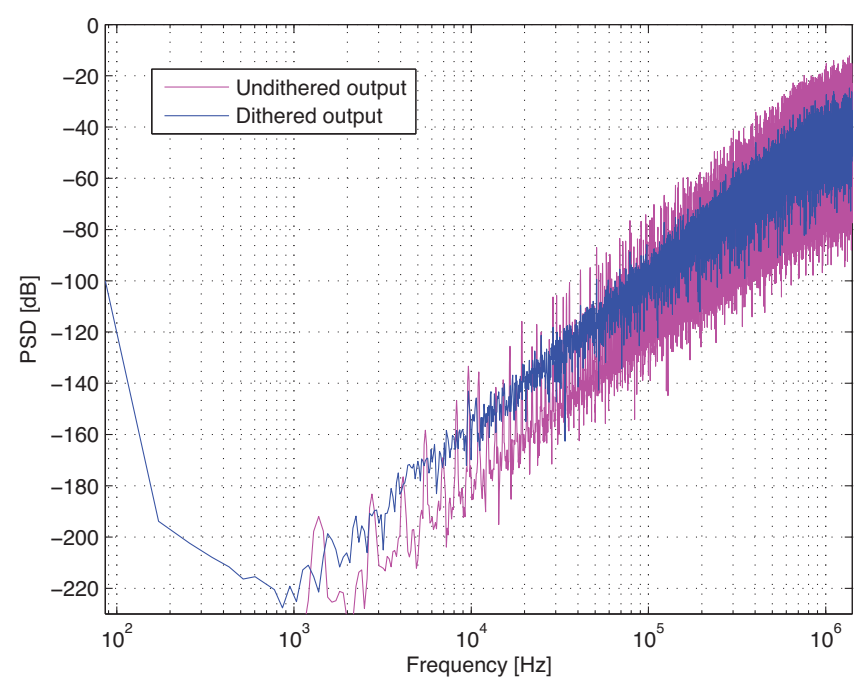

Fig. 2. Undithered and dithered MASH-111 simulated output spectrum.

dominant. Indeed, it is necessary, as outlined by equation (1), that the $\Sigma \Delta$ contribution is lower than other noise sources by at least $10-20 \mathrm{~dB}$. However, high order modulators are necessary because, with low order, the dither is not capable to spoil the repetitive patterns.

The design of single loop high-order modulators is difficult because of stability conditions. For this reason, the designer prefers using MASH architectures that obtain high order noise shaping by cascading first order modulators. Since the noise transfer function (NTF) of digital implementation is exactly what expected, the used architectures, made by common digital blocks such as accumulators, adders, and D flip flops, are convenient and effective. However, the output of MASH schemes is multi-bit. For a MASH-11 $\Delta N$ ranges from -1 to +2 , while for a MASH-111 $\Delta N$ varies from -3 to +4 . Therefore, the digital divider is complex and the power of the quantization error is larger.

Even with a MASH-111, the $\Sigma \Delta$ output spectrum is not completely tone-free. Fig. 2 shows a typical situation of an undithered output spectrum. To cancel out the tones, it is necessary to use dithering. It is the injection of small pseudorandom signals at the three inputs of the first order modulators. The pseudo-random sequence at the first integrator is white, the ones at the second and third are shaped by $\left(1-z^{-1}\right)$ and $\left(1-z^{-1}\right)^{2}$, respectively, to limit the in-band effect. The resulting spectrum looks like the second curve of Fig. 2.

This and other techniques have been used with good results. However, tones are not transformed into noise, but their amplitude is just reduced. Possible non-linearities of blocks used in the feedback loop cause mixing of tones that show up in the low frequency band as white floor. In some unfortunate case, the mixing generates a large component that is well visible in the output spectrum. The effect is outlined in Fig. 3 that shows the spectrum at the output of a MASH-111 and

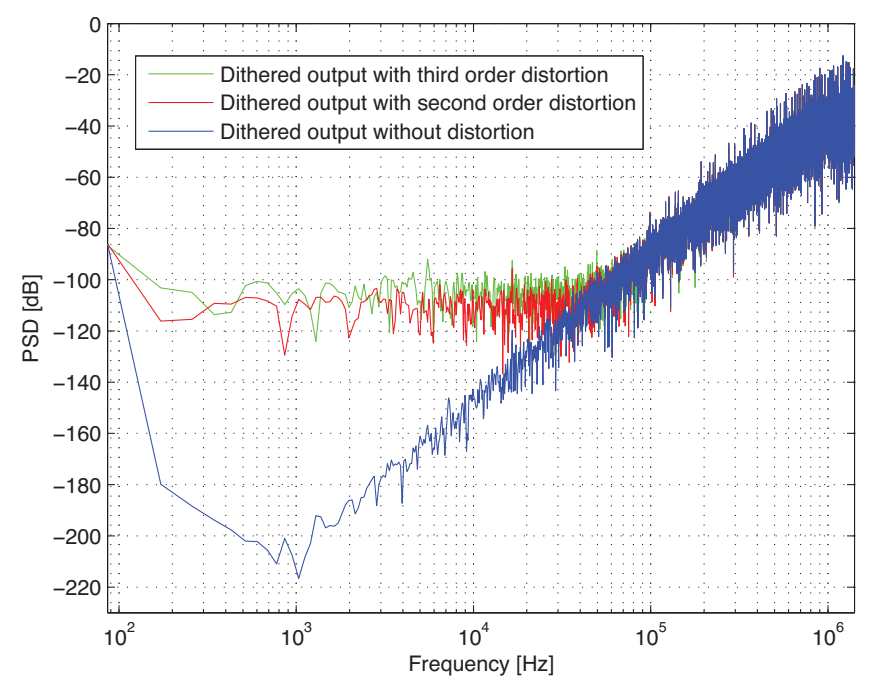

Fig. 3. Dithered MASH-111 output spectrum with and without distortion $\left(\alpha=2^{-15}, \beta=2^{-15}\right)$.

its processing with a non-linear block

$$
Y(t)=X(t)\left[1+\alpha X(t)+\beta X(t)^{2}\right] .
$$

The good spectrum at the output of the MASH-111 plus dithering at the three inputs degrades significantly with relatively small second order and third order distortion terms $\left(\alpha=2^{-15}, \beta=2^{-15}\right)$.

The obtained result leads to the following observations: high-order modulators are not fully effective in removing tones as the high-pass noise shaping of high-order modulators is corrupted by non linearity that cause an equivalent white noise.

\section{Proposed Method}

The dithering method foresees additive pseudo-random terms in critical points of the modulator. The method proposed here is to use multiplicative pseudo-random dithering that changes the coefficients used in the modulator architecture. As shown in Fig. 4 (a), the first order modulator adds the quantization error to the input. The proposed method foresees the change depicted in Fig. 4 (b). The quantization error is multiplied by a coefficient that changes randomly in time as given by

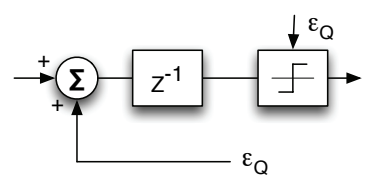

(a)

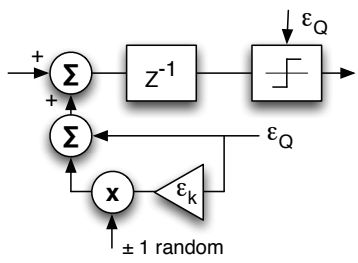

(b)

Fig. 4. Internal node of a conventional (a) and of the proposed (b) modulator. 


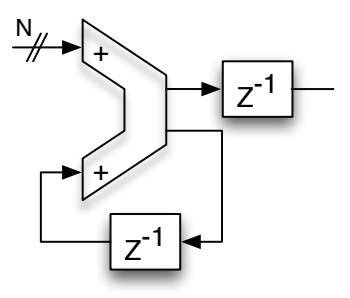

(a)

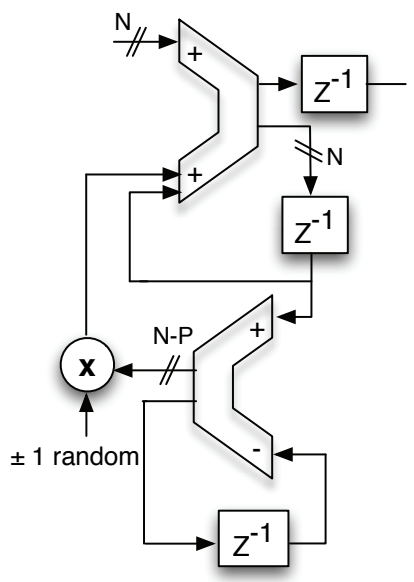

(b)

Fig. 5. Implementation for the schemes depicted in Fig. 4 (a) and (b).

$$
K(n T)=1+\epsilon_{K}(n T)
$$

where $\epsilon_{K}(n T)$ is a small convenient random number with constant module and random sign. The result is a sigmadelta with discrete-time variant coefficients. For digital sigmadelta modulators, the word-lenght of the added term must be quantized at the resolution of the accumulator. The operation just requires to truncate the dithered term or, eventually, by passing the truncated part through a first order digital $\Sigma \Delta$. Notice that the implementation of the time-variant architecture requires some additional hardware, as it is for the conventional dithering techniques. Fig. 5 (a) and (b) shows the implementations of the schemes of Fig. 4 (a) and (b), respectively.

Notice that the study of time variant $\Sigma \Delta$ modulators can not be done with the linearized method used for conventional $\Sigma \Delta$ architectures, but needs a special analysis similar to the one developed for time-variant filters. That study is not done in this work. The effectiveness of the method is proved by just simulations in the time-domain.

The scheme of Fig. 4 (b) can be the basis for the design of high order $\Sigma \Delta$ as, for example, the MASH-11 or the MASH111. Alternatively, it is possible to obtain second order or third order noise shaping with the schemes of Fig. 6. The inverse of the quatization error needs the following processing

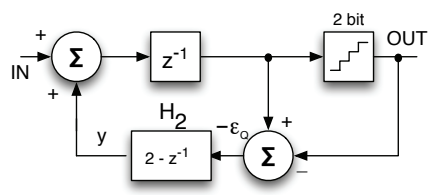

(a)

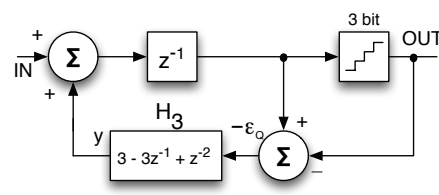

(a)

Fig. 6. Alternative block diagrams for second (a) and third (b) order $\Sigma \Delta$ modulators.

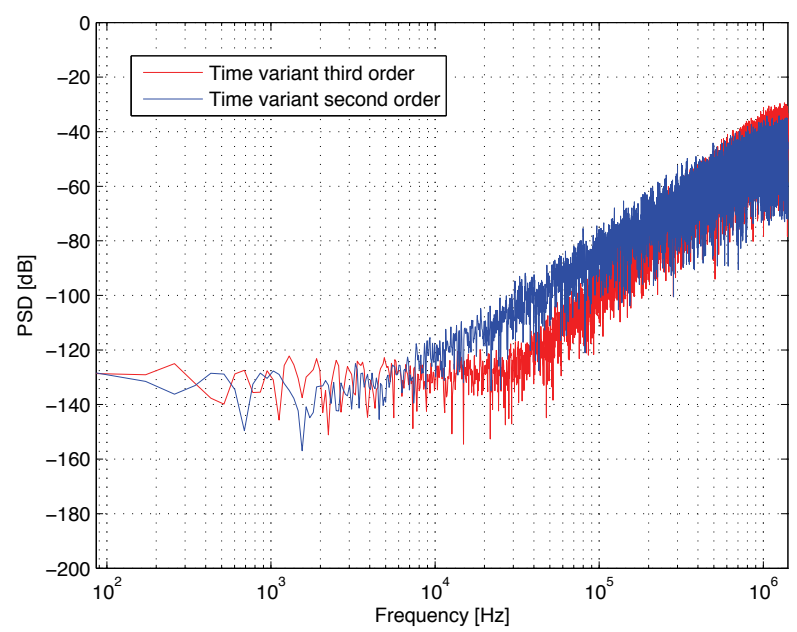

Fig. 7. Simulated output spectrum of the second and third order time variant $\Sigma \Delta$ modulator.

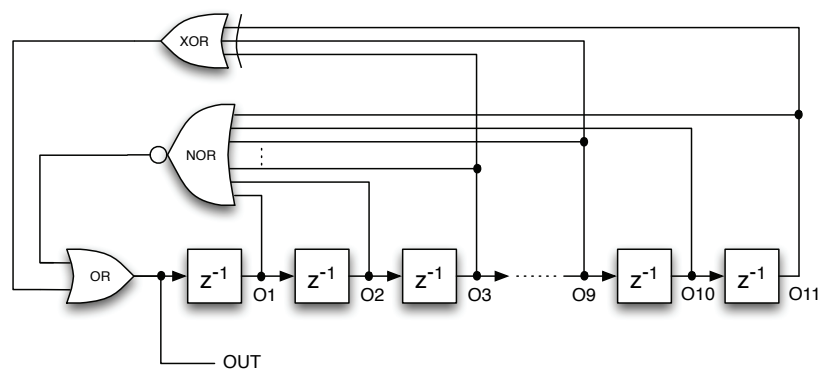

Fig. 8. Single-bit pseudo-random generator scheme.

$$
H_{2}=\left(2-z^{-1}\right) ; \quad H_{3}=\left(3-3 z^{-1}+z^{-2}\right)
$$

that identifies coefficients that can be possibly transformed into time variant by using dithering multipliers. The various multipliers used can have different amplitude and uncorrelated random signs.

Fig. 7 shows the output spectra of a time-variant second order and third order modulator with a critical value of constant input, used $\epsilon_{K}=1 / 2^{12}$ and with uncorrelated random signs. Our single-bit pseudo-random generator is made by a chain of 11 flip-flop with a suitable feedback logic that obtains a sequence $2^{11}$ long. The scheme is shown in Fig. 8. Uncorrelated sequences are given by using different taps of the inverters chain. The spectra of Fig.7 show that the using time variant coefficients causes a noise floor and reduces the noise amplification at high frequencies. The first effect is inessential, if the floor level is low. The second feature is beneficial for reducing the high frequency noise. Moreover, the obtained spectra are much more noise-like than the dithered counterparts.

A convenient test for verifying the method effectiveness is to pass the multi-bit output through a non-linear block with 


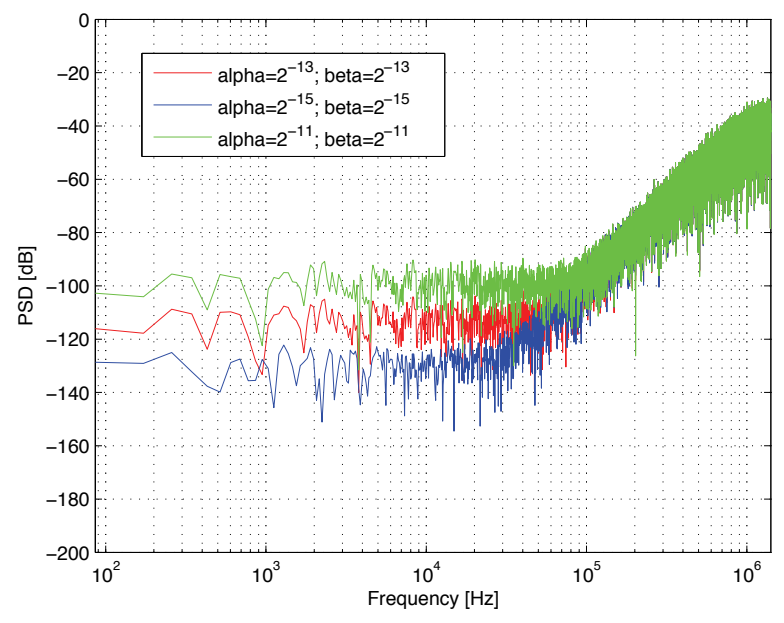

Fig. 9. Simulated spectrum of the third order time variant modulator with various distortion coefficients.

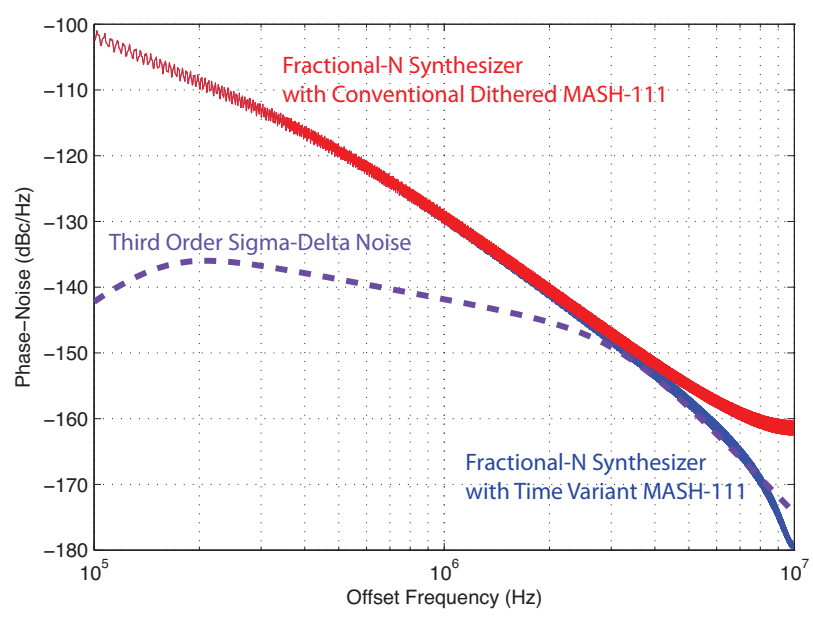

Fig. 10. Simulated phase noise of conventional dithered and time variant MASH-111 $\Sigma \Delta$ modulator.

response given by equation (2). The result is that the same coefficients $\left(\alpha=2^{-15}, \beta=2^{-15}\right)$ used in the simulation of Fig. 3 gives no noticeable changes in the time-variant MASH111, as shown in Fig. 9. To obtain noise floor above -140 $d B_{F S}$, it is necessary to use $\alpha=2^{-13}, \beta=2^{-13}$, a two orders of magnitude higher distortion. Fig. 9, shows that the noise floor of the conventional dithered MASH-111 is obtained with $\alpha$ and $\beta$ as large as $2^{-11}$.

\section{TIME-VARIANT $\Sigma \Delta$ IN Fractional-N Synthesizer}

The effective operation of the proposed $\Sigma \Delta$ architecture has been verified with behavioural simulation by its use in a fractional-N frequency synthesizer, [5-6]. The reference frequency is $25 \mathrm{MHz}$ and the fractional division number is 71.001953. The output frequency is $1.77504 \mathrm{GHz}$. Non linearity has been included in the loop. In particular, the charge

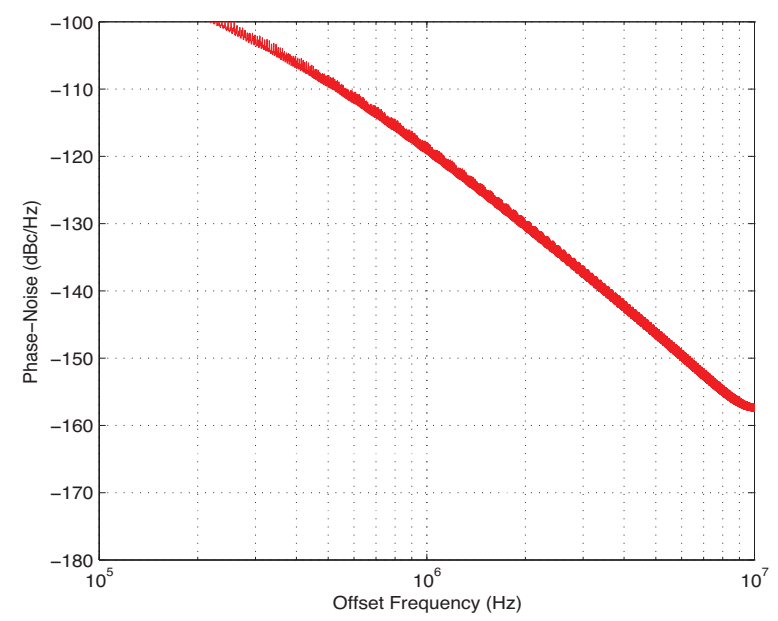

Fig. 11. Simulated phase noise of a time variant MASH-11 $\Sigma \Delta$ modulator.

pump has a dead zone of $4.5 \%$. The conventional dithered MASH-111 works as expected with linear components around the loop, but its performace worsens with the non-linearities. On the contrary, the time variant scheme does not experience any limit with the used non-linearities. Fig. 10 compares the two phase-noise plots. The phase noise in the ideal case is also shown.

The method is also effective with a MASH-II modulator for which the required $\Delta N(t)$ is half the one of the MASH-III. Fig. 11 shows that the simulated phase noise is clean with an expected higher level (about $10 \mathrm{~dB}$ ) because of a lower order in the noise shaping.

The obtained results minimally depend on the value of the fractional input. Therefore, depending on the system specifications, the designer can use affordable second or third order scheme with tone-free performance.

\section{ACKNOWLEDGMENT}

The authors thank FIRB, Italian National Program, Project RBAP06L4S5, for a partial financial support.

\section{REFERENCES}

[1] T. A. D. Riley, M. A Copeland and T. A. Kwasniewski, "Delta-Sigma Modulation in Fractional-N Frequency Synthesis", IEEE Journal of SolidState Circuits, vol. 28, no. 5, pp. 553-559, May 1993.

[2] W. Rhee and B.-S. Song, and A. Ali, "A 1.1-GHz CMOS Fractional-N Frequency Synthesizer with a 3-b Third Order $\Sigma \Delta$ Modulator", IEEE Journal of Solid-State Circuits, vol. 35, no. 10, pp. 1453-1460, October 2000.

[3] V. Friedman, "The Structure of the Limit Cycles in Sigma Delta Modulation", IEEE Trans. on Communications, vol. 36, pp. 972-979, August 1988.

[4] W. Chou and R. M. Gray, "Dithering and its effects on sigma-delta and multistage sigma-delta modulation", IEEE Trans. Inform. Theory, vol. 37, pp. 500-513, May 1991.

[5] Y. Fan, "Model, Analyze, and Simulate $\Sigma \Delta$ Fractional-N Frequency Synthesizers", Part 1 of 2 parts, Microwave \& RF, pp. 183-194, December 2000.

[6] M. H. Perrott, "Behavioral Simulation of Fractional-N Frequency Synthesizers and Other PLL Circuits", IEEE Design \& Test of Computers, pp. 74-83, July-August 2002. 